S.M. Yasir Arafat, ${ }^{1 \# *}$ M A Al Mamun, ${ }^{2,3 \# ~ M d . ~ S a l e h ~ U d d i n ~}$

\title{
Depression Literacy among First-Year University Students: A Cross-Sectional Study in Bangladesh
}

\author{
'Department of Psychiatry, Bangabandhu Sheikh Mujib Medical University, Dhaka, Bangladesh \\ 2Department of Public Health \& Informatics, Jahangirnagar University, Savar, Dhaka, Bangladesh \\ ${ }^{3}$ Undergraduate Research Organization \\ \#Both authors contributed equally to the paper. \\ *email: arafatdmc62agmail.com
}

DOI: 10.2478/gp-2019-0002

Received: 5 November 2018; Accepted: 22 November 2018

\begin{abstract}
Objectives: Early detection of depression has been assumed to lead to its earlier and better care. Increased depression literacy among the general population might play a vital role in the early and successful detection and treatment. We aimed to investigate depression literacy among the first year university students, to be able to compare their depression literacy with those of other previously investigated groups, we hypothesized that depression literacy might be different from other groups such as patients and other subjects from the general population. Knowledge about depression might also be different in female and male students.

Methods: This cross-sectional study was conducted among the first-year students of Jahangirnagar University, Bangladesh between January and May 2018. A convenience sample of 306 students was randomly identified from a list of students obtained from the authority. Relevant sociodemographic variables were collected. Students were interviewed using the Bangla Depression Literacy scale (D-Lit Bangla). This was a semi structured interview asking for the knowledge on 20 different characteristics of depression. Collected data were analysed to estimate the mean score and 95\% confidence intervals of D-Lit Bangla scores.

Results: The mean score of the correctly identified characteristics of depression was $6.55(95 \%$ CI $=6.31$ to 6.89$)$. Only five characteristics of depression were correctly identified by more than 50\% participants, that is, sleep change (76.7\%), feeling of guilt (75.2\%), low self-esteem (81.8\%), psychomotor changes (64.6\%) and identification of famous people suffering from depression (66.7\%). Only a very small portion of the participants correctly knew about psychotic symptoms and different options for the treatment of depression. There were no significant differences between male and female students in the amount and pattern of knowledge of depression.

Conclusions: The study revealed poor literacy status among the first-year university students of Bangladesh. Both male and female gender have similar amount of depression literacy.
\end{abstract}

\section{Keywords}

Depression literacy, Bangladesh, university students, depression.

\section{INTRODUCTION}

\section{Relevance and Symptoms of Depression}

Depression is a major mental disorder with a wide global distribution. Typically, it starts in early to mid-20s, it follows a relapsing and recurrent course and causes substantial impairments in multiple domains of life (Kessler \& Bromet 2013; World Health Organization 2017; American Psychiatric Association 2013). The World Health Organization (WHO) forecasts that depression will be the second most burdensome disease across the world by 2020 (Kessler \& Bromet 2013; WHO 2017).
Depressive episodes present with low mood, reduced pleasure in previously enjoyable activities, sleep problems, increase or decrease in appetite and weight, lack of energy, increased fatigability, attention and concentration problem, psychomotor agitation or retardation, undue feeling of guilts, suicidal thoughts, which may lead to suicides (Kessler \& Bromet 2013; WHO 2017; American Psychiatric Association 2013). Improving the knowledge of the population on depression might help detect depression early. 


\section{Health and Depression Literacy}

Health literacy (HL) was introduced first in 1974 and subsequently it has gained admiration in global research (Arafat et al. 2018a). Dan Nutbeam considered typologies of HL namely, functional health literacy, interactive health literacy, and critical health literacy (Nutbeam 2008).

HL has been considered as a fundamental element of patientcentred healthcare system and poor HL is a silent epidemic globally (Arafat et al. 2018a). It affects every aspect of health and it is well established that it plays a very important role in the identification of disorders, seeking help, initiatives to prevent, compliance of medication and so on (Arafat et al. 2017, 2018a).

Depression literacy (D-Lit) is an essential requirement to combat the disorder as it addresses the identification of depressive symptoms, receiving treatment options as well as making critical decisions during emergencies (Jeong et al. 2018; Arafat et al. 2018a). Moreover, it plays a contributory role in the treatment of depression as it is a chronic disorder (Arafat et al. 2018a,b; Arafat et al. 2017). Adequate literacy in young adults may affect disease care, help seeking behaviour, prevention of further complications and reduce the sufferings in the long term (Arafat et al. 2018a,b).

\section{Depression Literacy in Bangladesh}

Bangladesh is a thickly populated developing country in southeast Asia with recent significant enhancements in the health and literacy sectors. Nonetheless, HL has remained under addressed in the country (Arafat et al. 2018b). HL, mental health literacy, depression literacy are newer concepts in Bangladesh. Newer researches are gradually coming out to deal with the literacy issues among the general people, where the population have lower HL and frequent abnormal health perceptions (Arafat et al. 2017; Arafat \& Ahmed 2017; Arafat et al. 2018b). One previous comparative study reported that D-Lit was the highest among the medical graduates, followed by chronically ill patients, university graduates and depressed patients (Arafat et al. 2018b).

Without adequate health literacy, it is potentially impossible to get relevant relief from any specific disorder, which affects multiple domains of life chronically and somewhat silently. This includes depression. D-Lit Bangla is a tool to assess literacy on depression. It was validated by Arafat et al. in 2017 with the intention to assess depression literacy in Bangla speaking population (Arafat et al. 2017).
The present study aims to assess depression literacy among the first year students of a public university in Bangladesh. This will enable the comparison with other groups of the population who have been assessed with the same tool. We also hypothesized that the depression literacy is different by gender as this has been shown in other samples of the population.

\section{METHODS}

It was aimed to assess the depression literacy status among the first year university students in a public university of Bangladesh. We conducted face to face interviews with D-Lit Bangla to collect data on depression literacy.

Study Subjects: We selected the first-year university students who had completed 12 academic years. Respondents had basic education, were young, had not been in any specialized training, and thus, were unlikely to have an exposure to depression before.

Instrument and Assessment: Data were collected by administrating a semi-structured questionnaire comprising socio-demographic variables and D-Lit Bangla questionnaire. Sociodemographic variables included age, gender, marital status, residence, and name of the department of the respondents. D-Lit-Bangla consists of 20 items that specifically extract the knowledge about depression symptomatology, impact of depression and about its management.

The D-Lit Bangla was validated by Arafat et al. in 2017. The reliability of the D-Lit Bangla was measured by Cronbach's alpha, which was found to be 0.77 , reflecting acceptable internal consistency. Face validity, content validity and construct validity were assessed and found satisfactory at the time of validation of the instrument (Arafat et al. 2017). The instrument contains 20 questions about depression with three response options (yes, no, don't know) for each item. Single response from the three options of each item was indicated and recorded. Score '1' was given for each correct answer. Wrong and don't know answers were scored as ' 0 '. Total scores were calculated by summing up the scores of 20 items. Thus, the scores of D-Lit Bangla ranged from 0-20.

Design and Processes: This descriptive cross-sectional study was conducted among the first year students of Jahangirnagar University (JU), Dhaka, Bangladesh between January and May 2018. A total of 500 students were approached to be enrolled in the study. However, data were collected from a total of 306 students who provided written informed consent. Face to face interviews were performed to collect data at residential 
halls of the university. Initially, respondents were approached conveniently, consent was taken after explaining the study objectives and procedures. Following this, the main interview was conducted.

Data Analysis: After collection, the data were analysed using the Statistical Package for the Social Science version 16 (SPSS, Inc., Chicago, IL, USA) along with Microsoft Excel version 2010. Data were analysed on the basis of the objective of the study focusing the level of D-Lit and descriptive analysis was done. The literacy status was assessed in terms of the mean and standard deviation of the items as well as total scale. Cross tabulation was performed to assess the relationship of D-Lit in respect of gender by Chi-square test, where the level of significance was set at $p<0.05$.

Ethical Consideration: The study was approved from the ethical review committee of the department of Public Health \& Informatics of JU. Data were collected anonymously and an informed written consent was taken from the respondents before collecting the data. Confidentiality of data as maintained and any unauthorized exposer to data was not possible.

\section{RESULTS}

Sample Characteristics: Initially, five hundred students from the first year were approached, and finally, three hundred and six students participated in the study. Among the 306 respondents, $213(69.6 \%)$ were male and 93 (30.4\%) were female. Mean $( \pm$ SD) age of the respondents was $19.7( \pm 0.94)$ years ranging from 18 to 23 years.

Depression Literacy: The mean D-Lit score was 6.55 (95\% $\mathrm{CI}=6.31$ to 6.89 ), which ranged from $0-12$. The mean D-Lit score among males was 6.67 and among females it was 6.27. However, the difference was not statically significant, which was found by performing Student's t-test $(p=0.13)$. For the frequencies of correct responses for each item, see Table 1. It shows that very few items (5 questions) were correctly identified by more than $50 \%$ participants. Those item topics were as follows: sleep change (76.7\%), feeling of guilt (75.2\%), low selfesteem (81.8\%), psychomotor changes (64.6\%) and knowledge of depressed famous people $(66.7 \%)$. On the other hand, a very small portion of the participants correctly identified the items regarding the psychotic symptoms (reckless behaviour, irrelevant speech, auditory hallucination) and management of depression (Table1).

\section{DISCUSSION}

\section{Depression Literacy in Bangladeshi Students}

We aimed to evaluate the depression literacy status of a public university's fresher students in Dhaka, Bangladesh. The study revealed poor literacy status in recognition of the psychotic symptoms of depression as well as the treatment aspects of depression (Table 1). The mean score of D-Lit was 6.55 indicating a poor status in depression literacy among the students as the range of the scale is $0-20$.

As a country, in Bangladesh, university students are the prime educated part of a society who are supposed to lead the rest. Adequate literacy in depression among them is beneficial to identify, treat and also to make critical decisions regarding depression for themselves as well as many others in the society. On the contrary, low literacy would be responsible for deleterious effects on themselves, on society as well as on the country. The study revealed an alarming depression literacy status among young educated emerging leaders of Bangladesh, which demands appropriate attention.

\section{Comparison of the Knowledge about Depression with Other Groups}

One previous comparative study reported that D-Lit was the highest among the medical graduates (D-Lit mean:11.19), followed by chronically ill patients $(\mathrm{D}$-Lit mean $=9.59)$, private university graduates $(\mathrm{D}$-Lit mean $=8.34)$ and depressed patients $(\mathrm{D}$-Lit mean $=7.55)$ (Arafat et al. 2018b). The mean score of those 608 respondents was 9.19 measured by the same instrument, that is, D-Lit Bangla, with the same range, that is 0-20 (Arafat et al. 2018b). Medical graduates revealed to have the highest literacy status among the four groups. Nevertheless, the status should have more improved as they are supposed to have academic studies, as well as clinical exposure during their graduation. Depressed patients were found to have the least D-Lit status, which thus signifies the poor promotional efforts of clinicians towards their patients during clinical consultations. Surprisingly, D-Lit status of chronic physically ill patients who were not under clinical services for depression was more than the depressed patient group. There was no reasonable explanation for the finding. 
Table 1. Frequencies and percentages of correct responses on D-Lit Bangla items

\begin{tabular}{|c|c|c|}
\hline Item & Frequency & Percentage \\
\hline \multicolumn{3}{|l|}{ Biological Symptoms } \\
\hline Q6. Sleep disturbance (অতিরিক্ত ঘুমানো অথবা খুব কম ঘুমানো বিষন্নতার একটি লক্ষণ হতে পারে।) & 231 & $76.7 \%$ \\
\hline Q7. Change in eating habit (অতিরিক্ত খাওয়া বা রুচি কনে যাওয়া বিষন্নতার একটি লক্ষণ হতে পারে।) & 75 & $24.7 \%$ \\
\hline \multicolumn{3}{|l|}{ Cognitive Symptoms } \\
\hline Q2. Guilt feeling (বিষণ্ন মানুষ দোষ না করেও নিজেকে দোষী ভাবতে পারে।) & 227 & $75.2 \%$ \\
\hline Q4. Self-esteem (আত্মবিশ্বাসহীনতা এবং কম আত্মমর্যাদাবোধ বিষণ্নতার এর্কটি উপসর্গ হতে পারে।) & 248 & $81.8 \%$ \\
\hline Q8. Impaired memory and attention (বিষন্লতা আপনার স্যৃতিশক্তি এবং মনযোকের ব্যাঘাত ঘটায় না।) & 20 & $6.6 \%$ \\
\hline \multicolumn{3}{|l|}{ Behavioral symptoms } \\
\hline $\begin{array}{l}\text { Q9. Psychomotor changes (বিষন্নতার কারণে একজল মানুষের চলাফেরার গতি কমে } \\
\text { যেতে পারে বা চলাযেরায় চঞ্চলনতা দেখা যেতে পারে।) }\end{array}$ & 197 & $64.6 \%$ \\
\hline \multicolumn{3}{|l|}{ Psychotic symptoms } \\
\hline Q5. Auditory hallucination (বিষপ্নতায় থাকা মানুষ প্রায়শই এমন কণ্ঠ ক্নেত পারে যা আসটে অবাস্তব।) & 53 & $18.2 \%$ \\
\hline Q1. Irrelevant speech (বিষপ্ন মানুষ প্রায়ই অপরিকল্পিত ও এলোনেনো কথা বলে।) & 35 & $11.6 \%$ \\
\hline Q3. Reckless behavior (বেপরোয়া ব্যবহার বিষপ্নতার একটি সাধারণ লক্ষণ।) & 39 & $13 \%$ \\
\hline \multicolumn{3}{|l|}{ Impact of Depression } \\
\hline $\begin{array}{l}\text { Q11. Impairment due to mild depression (মাঝারি মাত্রার বিষন্লতার একজন } \\
\text { মানুমের জীবনকে হাপানি বা বধিরতার মতোই বিঘ্লিত করতে পারে।) }\end{array}$ & 114 & $38.0 \%$ \\
\hline Q12. Hospitalization (অধিকাংশ বিষন্ল মানুষকে হাসপাতালে ভর্তি হতে হয়।) & 135 & $45.8 \%$ \\
\hline Q13. Famous people (বহু বিখ্যাত ব্যাক্তি বিষন্লতায় ভুগেছেন।) & 202 & $66.7 \%$ \\
\hline \multicolumn{3}{|l|}{ Management } \\
\hline Q10. Role of psychologist (চিকিৎगা মনোবিজ্ঞানীগন বিষন্নতার ঔষধ দিতে পারেন।) & 66 & $22.3 \%$ \\
\hline $\begin{array}{l}\text { Q20. Antidepressants sedative property (गাধারণত বিষন্নতার সকল ঔষুধে } \\
\text { ঘুম বেশি হয় যার ফতে দিনের বেলায় কাজ করঢে অসুবিধা হতে পারে।) }\end{array}$ & 18 & $6.2 \%$ \\
\hline Q18. Antidepressants addictive property (বিষন্নতার, ঔষধ নেশা জাতীয়।) & 60 & $20.3 \%$ \\
\hline Q19. Duration of action (বিষন্নতার ঔষধ সাধারণত তৎক্ষণাৎ কাজ করে।) & 78 & $26.7 \%$ \\
\hline Q17. Timing of stopping antidepressants (বিষন্ন মানুষ ভালো বোধ করার সাটে সাথে ঔষধ বন্ধ করে দেওয়া উচিৎ।) & 41 & $14.1 \%$ \\
\hline $\begin{array}{l}\text { Q16. Vitamin's role (বিষন্নতার ক্ষেত্রে জীবনযাত্রা পরিবর্তন ও অন্যান্য চিকিৎসার } \\
\text { মধ্যে ভিটামিনইই সবচেয়ে উপকারী হতে পারে।) }\end{array}$ & 45 & $15.4 \%$ \\
\hline 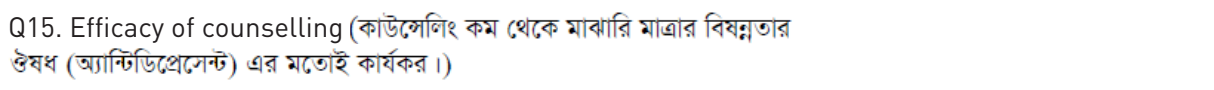 & 128 & $43.4 \%$ \\
\hline Q14. Other treatment options (বিষন্নতার অন্যান্য চিকিৎসা বিষন্নতার ঔষধের চেয়ে বেশি কার্যকর।) & 13 & $4.3 \%$ \\
\hline
\end{tabular}


A study in India revealed the mean D-Lit score 13.21 ( $\min =6$, $\max =19$ ), vis-à-vis the mean D-Lit score of the current study was $6.55(\min =0, \max =12)$, which indicates the poorer D-Lit status among the current study respondents (Ram et al. 2016). Among the undergraduate students in Sri Lanka, the ability to recognize depression was found to be low (Amarasuiya et al. 2015). In China, about $12 \%$ respondents were able to recognize depression in a given vignette (Wong et al. 2012). These differences can be explained by the different set-up of study, cultural construct of the respondents, different instruments, educational status of the respondents, educational system of the country, and the existing health promotional activities in the context.

\section{Gender Difference of Depression Literacy}

There was insignificant variation between the male and female students, which suggests that gender has a negligible role in depression literacy among the university students in Bangladesh. A study in India found that depression literacy was higher in females and it was positively associated with treatment compliance (Ram et al. 2016). Another study of British sample also revealed gender differences in health literacy status (Swami 2012). Previous comparative study in Bangladesh didn't address the gender issue (Arafat et al. 2018b).

\section{Consequences of Poor Depression Literacy}

Presently, adequate HL is a global prime concern (Arafat et al. 2018a), which is supported by a study in Portugal that reported low level of literacy in depression was well-thought-out as a key barrier for help seeking in mental health issues during the perinatal period (Fonseca et al. 2017). Lower knowledge in depression with higher levels of stigma was found to be associated with mental disorders; also, they can acts as barriers to seeking help from professionals (Kiropoulos et al. 2011).

This poor literacy status in depression, might be the representation of overall mental health literacy status which can be explained by absence of such resources in the academic curricula in the country in any stages of study focusing the MHL. However, improving literacy (health, mental health, depression and others) in adolescence is supposed to progress the later life as they are used to bear their changed behaviour across the life (Arafat et al. 2018a). Multiple school-based involvements covering physical and mental health have been researched and found to improve the literacy as well as quality of life (Arafat et al. 2018a). Basic literacy (health, mental health, depression) components should be coordinated into the academic curriculum with harmonious implementation of public mental health or mental health promotional activities in policy level can be a foresighted step to improve the grave situation (Arafat 2017). Along with adequate clinical care, regular provision of educative materials to the visiting patients should be considered in the clinical setting of a country like Bangladesh.

\section{LIMITATIONS}

A good proportion of students (response rate 61.2\%; nonresponse rate $38.8 \%$ ) refused to take part in the study that is a countable limitation of the current study. Students were reluctant to participate in the study. A token of appreciation could change the data collection in a positive direction. We faced financial constrains as it was a self-funded study with limited resources. Moreover, it was a cross-sectional study among a university first year students, which precludes to generalize the study results. Further, larger scale, longitudinal and interventional studies are necessary to generalize the observations.

\section{CONCLUSIONS}

The study revealed poor literacy status among the first-year university students of Bangladesh. Both male and female gender have similar status in depression literacy. The study suggests that urgent steps are warranted to improve depression literacy status among the specific group, which would reduce the sufferings of depression as well as to improve the quality of life.

\section{ACKNOWLEDGEMENTS}

Authors gratefully acknowledge the support of the Undergraduate Research Organization of Jahangirnagar University. Authors thank Prof. Reinhard Heun for his comments on language editing of the manuscript.

\section{CONFLICT OF INTEREST}

None to be declared.

\section{DECLARATION OF ETHICS}

The study was conducted complying with the declaration of Helsinki (1964). The study was approved from ethical review committee of the department of Public Health \& Informatics of JU. 


\section{DECLARATION OF INFORMED CONSENT}

Data were collected anonymously and informed written consent was taken from the respondents before collecting the data. Confidentiality of data was maintained and any unauthorized exposure to data was not possible.

\section{FUNDING}

Self-funded.

\section{REFERENCES}

American Psychiatric Association. Diagnostic and Statistical Manual of Mental Disorders, $5^{\text {th }}$ Edition, Washington DC: American Psychiatric Association, 2013;483.

Amarasuriya SD, Jorm AF, Reavley NJ. Quantifying and predicting depression literacy of undergraduates: a cross sectional study in Sri Lanka. BMC Psychiatry. 2015;15:13.

Arafat SMY. Mental health promotion or public mental health: the time demanded area. J Behav Heal. 2017;6(1):1-3.

Arafat SMY, Majumder MAA, Kabir R, Papadopoulos K, Uddin MS. Health literacy in school In: Papalois and Theodosopoulou, editor. Optimizing health literacy for improved clinical practices. Hershey, PA: Medical Information Science Reference; 2018a; 175-197.

Arafat SMY, Ahmed S, Uddin S. Depression literacy status in Bangladesh: a cross-sectional comparative observation. J Behav Heal. 2018b;7(2):91-97.

Arafat SMY, Shams SF, Chowdhury MHR, Chowdhury EZ, Hoque $M B$, Bari MA. Adaptation and validation of the Bangla version of the depression literacy questionnaire. J Psychiatry. 2017;20(4):412.

Arafat SMY, Ahmed S. Burden of misconception in sexual health care setting: a cross-sectional investigation among the patients attending a psychiatric sex clinic of Bangladesh. Psychiatry J. 2017;2017:9827083.

Fonseca A, Silva S, Canavarro MC. Depression literacy and awareness of psychopathological symptoms during the perinatal period. J Obstet Gynecol Neonatal Nurs. 2017;46(2):197-208.

Jeong YM, McCreary LL, Hughes TL. Qualitative study of depression literacy among Korean American parents of adolescents. JPN. 2018;56(1):48-56
Kessler RC, Bromet EJ. The epidemiology of depression across cultures. Annu Rev Public Health. 2013;18(34):119-138.

Kiropoulos LA, Griffiths KM, Blashki G. Effects of a multilingual information website intervention on the levels of depression literacy and depression-related stigma in Greek-born and Italianborn immigrants living in Australia: A randomized controlled trial. J Med Internet Res. 2011;13(2):e34.

Nutbeam D. The evolving concept of health literacy. Soc Sci Med. 2008;67(12):2072-2078.

Ram D, Benny N, Gowdappa B. Relationship between depression literacy and medication adherence in patients with depression. J Mood Disord. 2016;6(4):183-188.

Swami V. Mental health literacy of depression: gender differences and attitudinal antecedents in a representative British Sample. PLoS One. 2012;7(11):e49779.

Wong DFK, Xuesong H, Poon A, Lam AYK. Depression literacy among Chinese in Shanghai, China: a comparison with Chinesespeaking Australians in Melbourne and Chinese in Hong Kong. Soc Psychiatry Psychiatr Epidemiol. 2012;47(8):1235-1242.

World Health Organization. Depression and other common mental disorders: global health estimates. World Health Organization. 2017. 